\title{
Retrospective Comparison of Clinical and Angiographic Outcomes After Sirolimus-Eluting and Bare-Metal Stent Implantation in 312 Consecutive, Nonrandomized Severely Calcified Lesions Using a Rotablator
}

\author{
Takeyuki Kubota, ${ }^{1}$ MD, Tetsuya IshiKawa, ${ }^{1}$ MD, Yosuke Nakano, ${ }^{1}$ MD, Akira Endoh, ${ }^{1}$ MD, \\ Teruhiko Suzuki, ${ }^{1}$ MD, Hiroshi SaKamoto, ${ }^{1}$ MD, Toshio Hasuda, ${ }^{1}$ MD, Kamon Imai, ${ }^{1}$ MD, \\ Michihiro Yoshimura, ${ }^{2} \mathrm{MD}$, and Makoto MutoH, ${ }^{1} \mathrm{MD}$
}

\begin{abstract}
SUMMARY
In order to compare the long-term clinical and angiographic outcomes after sirolimus-eluting stent (SES) and baremetal stent (BMS) placement in severely calcified lesions using a rotablator under the widespread indication of SES, a nonrandomized examination of 312 consecutive lesions after successful implantation of a BMS (99 lesions in 84 patients; from January 2003) or SES (213 in 167; from September 2004) using a rotablator was conducted. The lesionbased primary endpoints (cardiac death and nonfatal recurrent myocardial infarction) and the secondary endpoint [binary restenosis (BR) (diameter stenosis > 50\%) at follow-up angiography] were retrospectively determined in August 2010. The incidence of primary endpoint in the SES group (2.3\%; mean follow-up period of $1289 \pm 526$ days) was significantly lower than that in the BMS group $(7.1 \% ; P=0.043 ; 1803 \pm 887$ days), although the several variables related to the endpoints were present in the SES group. Cox proportional hazard model analysis revealed that SES was not significantly related to a primary endpoint [hazard ratio of $0.42(95 \% \mathrm{CI}, 0.073-2.42 ; P=0.33)$ ]. The incidence of BR in the SES group $(21.3 \%)$ was not significantly different from that in the BMS group $(27.1 \%)(P=0.33)$. Multivariate logistic regression analysis revealed that SES was not a significant predictor of BR [Odds ratio of 0.78 (95\% CI, $0.41-1.51 ; P=$ $0.47)$ ]. Thus, although the results of the present retrospective nonrandomized study demonstrate the long-term safety of SES for calcified lesions using a rotablator in daily practice, SES did not show a benefit for the angiographic outcomes compared to BMS. (Int Heart J 2011; 52: 65-71)
\end{abstract}

Key words: Sirolimus-eluting stent, Calcification, Rotablator

A calcified coronary artery is a predictor of mortality ${ }^{1)}$ and is one of the complex and challenging targets of percutaneous coronary intervention (PCI). Since calcified coronary lesions are associated with the risk of suboptimal stent expansion, calcification of coronary arteries relates to in-stent restenosis (ISR) and target lesion revascularization $(\mathrm{TLR})^{2-4)}$ and subsequent stent thrombosis $(\mathrm{ST})^{5)}$ after sirolimuseluting stent (SES; Cypher Bx Velocity, Cordis, Johnson \& Johnson, NJ, USA) implantation. Therefore, it is necessary to evaluate whether SES is consistently beneficial for the treatment of calcified lesions in daily clinical practice with a widespread indication in the field of percutaneous coronary intervention (PCI). However, the impacts of severely calcified lesions on the long-term clinical and angiographic outcomes after successful SES placement in daily clinical practice are still not fully understood.

Rotational atherectomy (RA) using a rotablator (Boston Scientific, Natick, MA, USA) is useful for treating calcified lesions. The rotablator facilitates optimal stent implantation by debulking the hard calcified matrix and modifies vessel compliance. ${ }^{6}$ However, SES implantation with RA for a calcified coronary artery has yielded inconsistent outcomes, ${ }^{7-9)}$ despite the consistent efficacy revealed in the overall field of PCI using SES. ${ }^{2,10,11)}$

In the present study, we retrospectively examined whether SES implantation with RA improved clinical and angiographic outcomes and resolved calcified artery lesions in the more than 6 years since obtaining approval for the procedure. We performed a 7-year single-center nonrandomized study on 312 calcified lesions in 251 consecutive Japanese patients and retrospectively compared the long-term safety (clinical outcome) and efficacy (angiographic outcome) of SES and bare-metal stent (BMS) implantation with RA.

\section{MeTHODS}

Study design and population: The data were obtained from a

From the ${ }^{1}$ Department of Cardiology, Saitama Prefecture Cardiovascular Respiratory Center, Saitama and ${ }^{2}$ Department of Cardiology, The Jikei University School of Medicine, Tokyo, Japan.

Address for correspondence: Tetsuya Ishikawa, MD, 1696 Itai, Kumagaya, Saitama 360-0105, Japan.

Received for publication September 21, 2010.

Revised and accepted December 6, 2010. 
single-center cohort of patients who underwent PCI at the Saitama Prefecture Cardiovascular and Respiratory Center and were retrospectively investigated in August 2010. Between January 2003 and March 2010, we successfully treated 312 lesions in 251 patients with either a BMS or SES using RA from elective cases of de novo calcified stenotic lesions in native coronary artery with stable hemodynamics. Since the present study was undertaken to examine the impact of SES for se-

Table I. Baseline Lesion-Based Characteristics

\begin{tabular}{|c|c|c|}
\hline Lesion number & $\begin{array}{l}\text { BMS } \\
99\end{array}$ & $\begin{array}{l}\text { SES } \\
213\end{array}$ \\
\hline Age (years) & $71.5 \pm 9.45$ & $71.8 \pm 8.10$ \\
\hline Male gender $(\%)$ & 67.7 & 63.4 \\
\hline Diabetes $(\%)$ & 46.5 & 46.9 \\
\hline Hemodialysis $(\%)$ & 9.1 & 8.0 \\
\hline Prior myocardial infarction (\%) & 30.3 & 36.6 \\
\hline Prior coronary artery bypass graft $(\%)$ & 9.1 & 8.0 \\
\hline Low ejection fraction $(\%)$ & 5.1 & $12.2^{*}$ \\
\hline Ostial (\%) & 6.1 & 13.1 \\
\hline Bifurcation (\%) & 8.1 & $46.0^{* * *}$ \\
\hline Chronic total occlusion $(\%)$ & 7.1 & 5.2 \\
\hline Left anterior descending artery $(\%)$ & 61.6 & 64.8 \\
\hline Right coronary artery $(\%)$ & 28.3 & $17.8^{*}$ \\
\hline \multicolumn{3}{|l|}{ Procedure } \\
\hline IVUS use (\%) & 92.9 & 97.2 \\
\hline \multicolumn{3}{|l|}{ Rotablator } \\
\hline Number of burrs used & $1.24 \pm 0.43$ & $1.29 \pm 0.46$ \\
\hline Maximun burr size $(\mathrm{mm})$ & $1.76 \pm 0.21$ & $1.69 \pm 0.17$ \\
\hline $\begin{array}{l}\text { Maximun burr size : Reference vessel } \\
\text { diameter }\end{array}$ & $0.69 \pm 0.17$ & $0.68 \pm 0.15$ \\
\hline \multicolumn{3}{|l|}{ Stent } \\
\hline Number of stents per lesion & $1.31 \pm 0.62$ & $1.85 \pm 0.76^{* * *}$ \\
\hline Diameter of stent (mm) & $3.24 \pm 0.47$ & $3.06 \pm 0.36^{* * *}$ \\
\hline Length of stent (mm) & $30.3 \pm 17.3$ & $47.9 \pm 22.0^{* * *}$ \\
\hline Inflation pressure (atm) & $16.7 \pm 3.6$ & $20.4 \pm 3.0^{* * *}$ \\
\hline Bifurcation 2-stent technique (\%) & 0 & $11.7^{* * *}$ \\
\hline \multicolumn{3}{|l|}{ Quantitative coronary angiogram } \\
\hline \multicolumn{3}{|l|}{ Minimal lumen diameter (mm) } \\
\hline Preprocedure & $0.89 \pm 0.49$ & $0.95 \pm 0.52$ \\
\hline Postprocedure & $2.61 \pm 0.53$ & $2.44 \pm 0.47^{* *}$ \\
\hline Acute gain $(\mathrm{mm})$ & $1.74 \pm 0.62$ & $1.49 \pm 0.59^{* * *}$ \\
\hline \multicolumn{3}{|l|}{$\%$ diameter stenosis } \\
\hline Preprocedure & $66.4 \pm 17.7$ & $63.2 \pm 17.3$ \\
\hline Postprocedure & $10.0 \pm 12.7$ & $13.6 \pm 10.3^{*}$ \\
\hline \multicolumn{3}{|l|}{ Reference diameter (mm) } \\
\hline Preprocedure & $2.69 \pm 0.71$ & $2.61 \pm 0.58$ \\
\hline Postprocedure & $2.95 \pm 0.60$ & $2.85 \pm 0.53$ \\
\hline \multicolumn{3}{|l|}{ Lesion length (mm) } \\
\hline Preprocedure & $18.9 \pm 13.2$ & $21.7 \pm 15.7$ \\
\hline Primary endpoint $(n, \%)$ & $7,7.1$ & $5,2.3^{*}$ \\
\hline Death $(n, \%)$ & $7,7.1$ & $5,2.3$ \\
\hline Non-fatal re-MI $(n, \%)$ & 0 & 0 \\
\hline Definite stent thrombosis & 0 & 0 \\
\hline Observational duration (days) & $1803 \pm 887$ & $1289 \pm 526^{* * * *}$ \\
\hline
\end{tabular}

Characteristics related to patient, lesion, procedure, and QCA in BMS ( $n=$ $99)$ and SES $(n=213)$ were compared. Definitions are described in text. ${ }^{*} P<0.05,{ }^{* *} P<0.01$, and ${ }^{* * *} P<0.001$ versus BMS. verely calcified lesions using RA, patients who underwent stent implantation (BMS or SES) with RA who did not develop any periprocedural complications (ie, death, Q-wave myocardial infarction (MI), and emergency coronary artery bypass graft implantation), and in who postprocedural antegrade coronary flow was determined to be thrombolysis in myocardial infarction (TIMI) grade 3 flow with acceptable stent expansion as determined by angiography and intravascular ultrasonography (IVUS) were enrolled. One patient with recent inferior MI died due to cardiac rupture in the inferior area after SES implantation to the mid portion of the left anterior descending artery (LAD). Four patients developed slow flow phenomenon (TIMI grade 1 flow) during the RA procedures prior to BMS or SES implantation. Three patients developed coronary perfo-

Table II. Predictors of Primary Endpoints After SES and BMS Implantations for Severely Calcified Lesions With RA

\begin{tabular}{lccc}
\hline & Hazard ratio & $95 \% \mathrm{CI}$ & $P$ \\
\hline Hemodialysis & 19.2 & $2.46-149$ & 0.005 \\
Age & 1.16 & $1.04-1.29$ & 0.006 \\
$:$ & $:$ & $:$ & $:$ \\
SES & 0.421 & $0.073-2.42$ & 0.333 \\
$:$ & $:$ & $:$ & $:$ \\
\hline
\end{tabular}

Predictors of primary endpoints were analyzed by the Cox proportional hazard model for all 312 lesions. SES was not related to the risk of a primary endpoint. Definitions are described in text.

Table III. Baseline Characteristics of Lesions Followed-Up Angiographically

\begin{tabular}{|c|c|c|}
\hline $\begin{array}{c}\text { Lesion number } \\
\text { Interval until secondary angiogram } \\
\text { (days) }\end{array}$ & $\begin{array}{c}\text { BMS } \\
70 \\
262 \pm 218\end{array}$ & $\begin{array}{c}\text { SES } \\
164 \\
494 \pm 333^{* * * *}\end{array}$ \\
\hline Age (years) & $70.3 \pm 9.0$ & $70.2 \pm 8.0$ \\
\hline Male gender $(\%)$ & 70.0 & 65.9 \\
\hline Diabetes (\%) & 51.4 & 47.0 \\
\hline Hemodialysis $(\%)$ & 10.0 & 7.9 \\
\hline Prior myocardial infarction $(\%)$ & 34.3 & 38.4 \\
\hline Prior coronary artery bypass graft $(\%)$ & 12.9 & 7.3 \\
\hline Low ejection fraction $(\%)$ & 5.7 & 9.1 \\
\hline Ostial (\%) & 5.7 & 14.6 \\
\hline Bifurcation (\%) & 7.1 & $46.3^{* * * *}$ \\
\hline Chronic total occlusion (\%) & 5.7 & 6.1 \\
\hline Left anterior descending artery $(\%)$ & 58.6 & 65.2 \\
\hline Right coronary artery $(\%)$ & 32.9 & $20.1^{*}$ \\
\hline IVUS use (\%) & 97.1 & 98.8 \\
\hline Number of burrs used & $1.21 \pm 0.41$ & $1.33 \pm 0.48$ \\
\hline Maximun burr size $(\mathrm{mm})$ & $1.76 \pm 0.21$ & $1.70 \pm 0.18^{*}$ \\
\hline $\begin{array}{l}\text { Maximun burr size : Reference vessel } \\
\text { diameter }\end{array}$ & $0.70 \pm 0.18$ & $0.67 \pm 0.15$ \\
\hline Number of stents per lesion & $1.31 \pm 0.63$ & $1.88 \pm 0.77^{* * * *}$ \\
\hline Diameter of stent (mm) & $3.27 \pm 0.50$ & $3.08 \pm 0.35^{* *}$ \\
\hline Length of stent (mm) & $30.5 \pm 17.2$ & $49.0 \pm 22.3^{* * * *}$ \\
\hline Inflation pressure (atm) & $16.4 \pm 3.5$ & $20.4 \pm 2.9^{* * *}$ \\
\hline Bifurcation 2-stent technique (\%) & 0 & $11.0^{* *}$ \\
\hline
\end{tabular}

Characteristics related to patient, lesion, procedure, and QCA in the cohort of lesions followed-up angiographically treated by BMS $(n=70)$ and SES $(n=164)$ were compared. Definitions are described in text. ${ }^{*} P<0.05,{ }^{* *} P$ $<0.01$, and ${ }^{* * * *} P<0.001$ versus BMS. 
ration immediately after balloon inflation, and BMS or SES implantation implicated in Q-wave MIs. These 8 cases were excluded. The number of cases with complications in periprocedural non-Q wave MI was not fully clarified, because peak serum levels of myocardial creatine kinase isoenzyme and/or that of troponin $\mathrm{T}$ were not continuously measured if the symptoms, electrocardiogram, and antegrade epicardial coronary flow were acceptable. Accordingly, 99 lesions in 84 patients with BMS (BMS group) and 213 lesions in 167 patients with SES (SES group) were included. After SES approval in August 2004, more patients received SES (91.4\%) than the 18 patients (20 lesions) treated with BMS (but was dependent upon the doctor's discretion). Approximately 6-7\% of the lesions were treated by RA at our institute per year.

The angiographic outcomes of the follow-up angiogram (fu-CAG) examination up to August 2010 were included in this study. The fu-CAG examination was planned for at approximately 6-12 months for the BMS group and approximately for at 10-18 months for the SES group. The percentage of lesions that underwent fu-CAG examination in the BMS group and SES group was $70.7 \%$ and $77.0 \%$, respectively (no significant change; $P=0.23$ ).

Procedure and antiplatelet therapy: All patients were informed of the necessity for the ablation with RA for calcified lesions, and their consent was obtained. RA was performed in the following cases: (i) when it was not possible to insert devices (small sized balloon and/or IVUS device), and (ii) when the balloon used for predilation of the target lesion was indented. Burr size in the present nonrandomized study was determined by the operator; the maximum burr size to reference vessel diameter ratio calculated by dividing the maximum burr size by the preprocedural reference diameter (defined below) was approximately $0.70 .{ }^{12)}$ (Table I)

Table IV. Angiographic Outcomes of Angiographic Followed-Up Lesions

\begin{tabular}{|c|c|c|}
\hline Lesion number & $\begin{array}{c}\text { BMS } \\
70\end{array}$ & $\begin{array}{c}\text { SES } \\
164\end{array}$ \\
\hline \multicolumn{3}{|l|}{ Minimal lumen diameter (mm) } \\
\hline Preprocedure & $0.93 \pm 0.50$ & $0.97 \pm 0.55$ \\
\hline Postprocedure & $2.62 \pm 0.55$ & $2.46 \pm 0.46^{*}$ \\
\hline Follow-up & $1.60 \pm 0.68$ & $1.99 \pm 0.76^{* * * *}$ \\
\hline \multicolumn{3}{|l|}{$\%$ diameter stenosis } \\
\hline Preprocedure & $63.7 \pm 17.6$ & $63.7 \pm 17.5$ \\
\hline Postprocedure & $10.6 \pm 11.9$ & $13.8 \pm 10.0$ \\
\hline Follow-up & $38.6 \pm 22.0$ & $32.9 \pm 22.4$ \\
\hline \multicolumn{3}{|l|}{ Reference diameter (mm) } \\
\hline Preprocedure & $2.69 \pm 0.78$ & $2.67 \pm 0.58$ \\
\hline Postprocedure & $2.99 \pm 0.60$ & $2.87 \pm 0.54$ \\
\hline Follow-up & $2.56 \pm 0.67$ & $2.98 \pm 0.59^{* * * *}$ \\
\hline \multicolumn{3}{|l|}{ Lesion length (mm) } \\
\hline Preprocedure & $18.8 \pm 12.2$ & $22.0 \pm 15.7$ \\
\hline Follow-up & $15.0 \pm 10.3$ & $6.6 \pm 6.3^{* * * *}$ \\
\hline \multicolumn{3}{|l|}{ Net value } \\
\hline Acute gain $(\mathrm{mm})$ & $1.69 \pm 0.62$ & $1.49 \pm 0.61^{*}$ \\
\hline Late luminal loss (mm) & $1.01 \pm 0.64$ & $0.47 \pm 0.74^{* * * *}$ \\
\hline Binary restenosis $(\%)(\% \mathrm{DS}>50)$ & 27.1 & 21.3 \\
\hline Type II-IV ISR $(\%)$ & 73.7 & $45.7^{*}$ \\
\hline Severe restenosis $(\% \mathrm{DS}>=70)$ & 10.0 & 9.8 \\
\hline
\end{tabular}

Angiographic outcomes with QCA analysis in BMS $(n=70)$ and SES $(n=164)$ were compared. Definitions are described in text. ${ }^{*} P<0.05$ and **** $P<0.001$ versus BMS.
During the procedure, we used IVUS to ascertain optimal expansion and apposition of the stents. ${ }^{13,14)}$ In addition, the stents were placed to fully cover the target with assistance from angiographic and/or intravascular IVUS data. A microcatheter was used to facilitate the procedure. ${ }^{15)}$ High-pressure ballooning using a noncompliant balloon ${ }^{5,16)}$ was performed after stent implantation.

In addition to aspirin, ticlopidine (200 mg/day) was administered for at least 2 weeks in the BMS group and at least 12 weeks in the SES group patients, however, the duration of dual-antiplatelet therapy was decided by the doctor.

Endpoints: Primary endpoint-related clinical outcomes were death, including death due to unknown reasons, and nonfatal recurrent MI. Secondary endpoint-related angiographic outcome was in-stent binary restenosis (BR), which was defined as $>50 \%$ diameter stenosis (DS) at fu-CAG. Both endpoints were lesion-based.

Estimated variables: The definitions of the variables used as baseline characteristics were as follows: (1) patient; clinical, lesion, and procedure at index procedure, age (age at primary stenting); male gender; diabetes (patients with diabetes mellitus); low ejection cardiac dysfunction (ejection fraction of left ventricle less than 40 as evaluated by ultrasonography or left ventriculogram); location of the culprit lesion in the LAD artery and right coronary artery (RCA); IVUS use (availability of IVUS during PCI); ostial (ostial lesions in 4 native coronaries); bifurcation (bifurcative lesions requiring any treatment of the side branch); chronic total occlusion (total occlusion more than 3 months; the previous 3 variables have been defined according to the American College of Cardiology/American Heart Association classification of lesions); number of burrs used (number of burrs used per lesion); maximum burr size (maximum diameter of the burr used to ablate); maximum burr size : reference vessel diameter (defined as above); number of stents per lesion (number of implanted stents per lesion); stent diameter (maximum diameter of the balloon used to dilate the stent); stent length (length of the stented segment, calculated by adding the length of each stent, regardless of overlap); inflation pressure (maximum pressure at the maximum inflation diameter of the balloon); bifurcation 2-stent technique (performing any bifurcation 2-stent techniques during the procedure): definite stent thrombosis (defined by the Academic Research Consortium) ${ }^{17)}$ : observational duration (duration in days until censored after index procedure).

The quantitative coronary angiography (QCA) parameters were measured using the test circulatory system (TCS) network system according to a previously described method. ${ }^{18)}$ The values of minimal lumen diameter (MLD), \% DS, reference diameter (RD), and lesion length were obtained (CAAS-2 system; Netherlands) at 3 points: before PCI (preprocedural), immediately after successful PCI (postprocedural), and at chronic phase (during follow-up). In addition, we calculated acute gain (postprocedural MLD minus preprocedural MLD) and late luminal loss (postprocedural MLD minus MLD at chronic phase). BR was defined as $>50 \%$ DS at fu-CAG. Merhen, et $a l^{19)}$ reported that ISR could be type 1 or focal type (lesion length at chronic phase $\geq 10 \mathrm{~mm}$ ) and types 2-4 or diffuse type $(<10 \mathrm{~mm})$. The ratio of type $2-4$ ISR in the SES group was compared with that in the BMS group. In addition, severe in-stent restenosis as $\geq 70 \%$ DS at fu-CAG was also compared. Statistics: Baseline characteristic variables are expressed as 
the mean \pm standard deviation (SD). The variables of the BMS and SES groups were compared using unpaired $t$-tests for continuous values and $\chi^{2}$ tests for categorical values. The Cox proportional hazard model was used to analyze the risk variables of the lesion-based primary endpoint by including the 24 baseline variables shown in Table I. Multiple logistic regression analysis was performed to analyze the predictors of BR by including the same 24 baseline variables. The baseline variables and the incidence of BR in the SES group were compared with those in the BMS group, after selecting the similar length of stent with a similar diameter of stent in the angiographic follow-up lesions from the BMS and SES groups (Tables III and IV). Statistical analyses were performed using Windows version 8 of Stata software (StataCorp, College Station, TX, USA), and the difference was considered significant when the $P$ was less than 0.05 .

\section{RESUlts}

Baseline lesion-based characteristics and primary endpoints: We compared 29 variables related to the patients, lesions, procedures, and quantitative coronary angiograms (QCA) of the SES group with those of the BMS group (Table I). The percentage of low ejection fraction in the SES group was significantly higher than that in the BMS group $(P<0.05)$. In the lesion characteristics, the percentage of bifurcated lesions in the SES group was significantly higher than that in the BMS group $(P<0.001)$. The location of the RCA in the SES group was significantly lower than that in the BMS group $(P<0.05)$. All 5 variables related to stent implantation in the SES group were significantly different from those of the BMS group (all were $P<0.001)$. Among the QCA variables, postprocedural MLD and RD and acute gain in the SES group were significantly different from those in the BMS group $(P<0.01,0.05$, and 0.001 , respectively).

The incidences of primary endpoints in the SES group $(2.3 \%)$ were significantly lower than that in the BMS group $(7.1 \%)(P<0.05)$. The incidence of nonfatal recurrent MI was 0 in both groups.

There was no definite stent thrombosis (ST) in either group. The mean observational duration was significantly shorter in the SES group than in the BMS group $(P<0.001)$.

Predictors of primary endpoints: Cox proportional hazard model analyses revealed that hemodialysis [hazard ratio of $19.2 ; 95 \%$ CI, 2.46-149; $P=0.005]$ and age $(1.16 ; 1.04-1.29$; $P=0.006)$ were significant predictors of primary endpoints in all 312 lesions. SES was not related to the risk of a primary endpoint (0.421; 0.073-2.42; $P=0.333$ ) (Table II).

Baseline characteristics of angiographic follow-up lesions: The lesion-related baseline variables assessed in fu-CAG are presented in Table III. The mean interval until fu-CAG in the SES group was significantly longer than that in the BMS group ( $P$ $<0.001)$. The percentages of bifurcation and RCA in the SES group were significantly different from those in the BMS group $(P<0.001$ and $P<0.05$, respectively). The maximum burr size in the SES group was significantly smaller than that in the BMS group $(P<0.05)$. As shown in Table I, the differences in 5 stent-related variables were highly significant between the two groups $(P<0.001$ or $<0.01)$.

Angiographic outcomes and secondary endpoint: The angiographic outcomes in the SES $(n=164)$ and BMS $(n=70)$ groups are shown in Table IV. The mean values of postprocedural, follow-up MLD, and acute gain in the SES group were significantly different from those in the BMS group $(P<0.05$, $<0.001$ and $<0.001$, respectively). The mean values of followup RD and lesion length in the SES group were significantly different from those in the BMS group $(P<0.001$, and $<$ 0.001 , respectively). The mean value of late luminal loss in the SES group was significantly smaller than that in the BMS group $(P<0.001)$.

The incidence of BR in the SES group was not significantly different from that in the BMS group. The percentage of type II-IV ISR in the SES group was significantly lower than that in the BMS group $(P<0.05)$. The incidence of severe restenosis in the SES group was not significantly different from that in the BMS group.

Predictors of binary restenosis: Multivariate logistic regression analysis revealed that hemodialysis [Odds ratio of 3.09; $95 \%$ CI, 1.10-8.70; $P=0.032$ ] was the single predictor of BR, although age was the predictor in univariate analysis. SES [0.784; 0.408-4.51; $P=0.465]$ was not a significant predictor of BR (Table V). Stent length [1.000; 0.987-1.01; $P=0.918$ ] was not a significant predictor of BR (data not shown in Table V).

Comparison of angiographic outcomes after selecting for similar stent length with similar stent diameter in the BMS and SES groups: The lesion-related baseline variables and angiographic outcomes after selecting for similar stent length with a similar stent diameter in the 46 lesions followed-up angiographically in the BMS and SES groups are shown in Table VI. The mean interval until fu-CAG in the SES group was significantly longer than that in the BMS group $(P<0.001)$. The percentage of CTO in the SES group was significantly different from that in

Table V. Predictors of Binary Restenosis

\begin{tabular}{lcccccc}
\hline & \multicolumn{3}{c}{ Univariate analysis } & \multicolumn{4}{c}{ Multivariate analysis } \\
& Odds ratio & $95 \%$ CI & $P$ & Odds ratio & $95 \%$ CI & $P$ \\
& & & & & \\
Hemodialysis & 3.98 & $1.56-10.2$ & 0.004 & 3.09 & $1.10-8.70$ & 0.032 \\
Age & 0.961 & $0.928-0.996$ & 0.030 & 0.978 & $0.940-1.02$ & 0.263 \\
$:$ & $:$ & $:$ & $:$ & & & \\
SES & 0.784 & $0.408-1.51$ & 0.465 & & & \\
$:$ & $:$ & $:$ & $:$ & & & \\
\hline
\end{tabular}

Predictors of binary restenosis were analyzed by logistic regression analysis in all 234 lesions followed-up angiographically. SES was not a significant predictor of binary restenosis. Definitions are described in text. 
the BMS group $(P<0.05)$. The mean inflation pressure in the SES group was significantly higher than that in the BMS group $(P<0.001)$. The mean values of preprocedural MLD and lesion length, and follow-up MLD, RD, and lesion length in the SES group were significantly different from those in the BMS

Table VI. Baseline Characteristics and Angiographic Outcomes of 46 Lesions Followed-Up Angiographically and Treated With BMS or SES of Similar Stent Length and Stent Diameter

\begin{tabular}{|c|c|c|}
\hline $\begin{array}{l}\text { Lesion number } \\
\text { Interval until secondary angiogram } \\
\text { (days) }\end{array}$ & $\begin{array}{c}\text { BMS } \\
46 \\
280 \pm 232\end{array}$ & $\begin{array}{c}\text { SES } \\
46 \\
484 \pm 344^{* * * *}\end{array}$ \\
\hline Age (years) & $70.7 \pm 10.0$ & $70.3 \pm 7.8$ \\
\hline Male gender $(\%)$ & 69.6 & 67.4 \\
\hline Diabetes $(\%)$ & 50.0 & 39.1 \\
\hline Hemodialysis $(\%)$ & 2.2 & 6.5 \\
\hline Prior myocardial infarction (\%) & 39.1 & 41.3 \\
\hline Prior coronary artery bypass graft $(\%)$ & 10.9 & 10.9 \\
\hline Low ejection fraction $(\%)$ & 2.2 & 2.2 \\
\hline Ostial (\%) & 4.3 & 8.7 \\
\hline Bifurcation $(\%)$ & 28.3 & 45.7 \\
\hline Chronic total occlusion (\%) & 8.7 & $0^{*}$ \\
\hline Left anterior descending artery $(\%)$ & 65.2 & 54.3 \\
\hline Right coronary artery $(\%)$ & 28.3 & 28.3 \\
\hline IVUS use (\%) & 97.8 & 100.0 \\
\hline Number of burrs used & $1.24 \pm 0.43$ & $1.37 \pm 0.53$ \\
\hline Maximun burr size (mm) & $1.72 \pm 0.19$ & $1.73 \pm 0.16$ \\
\hline $\begin{array}{l}\text { Maximun burr size : Reference vessel } \\
\text { diameter }\end{array}$ & $0.70 \pm 0.17$ & $0.67 \pm 0.14$ \\
\hline Number of stents per lesion & $1.37 \pm 0.57$ & $1.33 \pm 0.52$ \\
\hline Diameter of stent (mm) & $3.07 \pm 0.38$ & $3.06 \pm 0.37$ \\
\hline Length of stent (mm) & $33.2 \pm 14.4$ & $32.7 \pm 14.1$ \\
\hline Inflation pressure (atm) & $16.4 \pm 3.3$ & $20.1 \pm 3.4^{* * * *}$ \\
\hline Bifurcation 2-stent technique (\%) & 0 & 6.5 \\
\hline \multicolumn{3}{|l|}{ Quantitative coronary angiography } \\
\hline \multicolumn{3}{|l|}{ Minimal lumen diameter (mm) } \\
\hline Preprocedure & $0.87 \pm 0.46$ & $1.08 \pm 0.45^{*}$ \\
\hline Postprocedure & $2.54 \pm 0.51$ & $2.49 \pm 0.49$ \\
\hline Follow-up & $1.53 \pm 0.67$ & $2.04 \pm 0.83^{* *}$ \\
\hline \multicolumn{3}{|l|}{$\%$ diameter stenosis } \\
\hline Preprocedure & $64.2 \pm 18.0$ & $59.2 \pm 15.5$ \\
\hline Postprocedure & $10.5 \pm 12.7$ & $12.5 \pm 9.91$ \\
\hline Follow-up & $39.0 \pm 22.7$ & $32.5 \pm 23.6$ \\
\hline \multicolumn{3}{|l|}{ Reference diameter (mm) } \\
\hline Preprocedure & $2.59 \pm 0.68$ & $2.68 \pm 0.57$ \\
\hline Postprocedure & $2.93 \pm 0.60$ & $2.86 \pm 0.53$ \\
\hline Follow-up & $2.44 \pm 0.64$ & $3.06 \pm 0.63^{* * *}$ \\
\hline \multicolumn{3}{|l|}{ Lesion length (mm) } \\
\hline Preprocedure & $22.2 \pm 13.5$ & $17.0 \pm 11.5^{*}$ \\
\hline Follow-up & $14.7 \pm 10.8$ & $6.27 \pm 6.99^{* * * *}$ \\
\hline \multicolumn{3}{|l|}{ Net value } \\
\hline Acute gain $(\mathrm{mm})$ & $1.68 \pm 0.62$ & $1.40 \pm 0.54^{*}$ \\
\hline Late luminal loss (mm) & $1.02 \pm 0.65$ & $0.45 \pm 0.85^{* * *}$ \\
\hline Binary restenosis $(\%)(\% \mathrm{DS}>50)$ & 30.4 & 19.6 \\
\hline Type II-IV ISR (\%) & 19.6 & 8.7 \\
\hline Severe restenosis $(\% \mathrm{DS}>=70)$ & 8.7 & 10.9 \\
\hline
\end{tabular}

By selecting for the lesions followed-up angiographically and treated by the similar stent diameter and stent length among BMS $(n=46)$ and SES ( $n=46$ ) groups, characteristics related to patient, lesion, procedure, and QCA were compared. Definitions are described in text. ${ }^{*} P<0.05,{ }^{* *} P<$ 0.01 , and ${ }^{* * *} P<0.001$ versus BMS. group $(P<0.05,<0.05,<0.01,<0.001$ and $<0.001$, respectively). The mean values of acute gain and late luminal loss in the SES group were significantly smaller than those in the BMS group $(P<0.05$, and $<0.001$, respectively $)$.

The incidence of BR in the SES group was not significantly different from that in the BMS group $(P=0.23)$. The percentage of type II-IV ISR in the SES group (8.7\%) was not significantly different from that in the BMS group $(P=0.13)$. The incidence of severe restenosis in the SES group was not significantly different from that in the BMS group.

\section{Discussion}

We undertook this retrospective study with the aim of evaluating the long-term safety and efficacy of SES, particularly for severely calcified lesions using RA, used with the wideranging indication of PCI in daily clinical practice in the current drug-eluting stent (DES) era. This was because severely calcified coronary lesions are usually observed in older patients with multivessel diseases, diffuse atherosclerotic lesions, and cardiac dysfunction ${ }^{6,20,21)}$ and are consistent predictors of cardiac events, including stent thromboses. ${ }^{1)}$ Thus, although the incidence of stent thrombosis (ST) defined by the Academic Research Consortium $^{17)}$ is significantly less in Japan than in western counties, ${ }^{3,22,23)}$ it was necessary to establish the longterm safety of SES implantation compared to BMS for severely calcified lesions using RA in Japanese patients. In the present historical comparison, the SES group exhibited many predictors of primary and secondary endpoints and ST reflecting the widespread indication (Tables I, III); the predictors were as follows: low ejection fraction, ${ }^{22,24,25)}$ longer and larger number of stents but smaller stent diameter, ${ }^{26,27}$ and bifurcation and bifurcation 2-stent technique. ${ }^{3,22,25,28)}$ However, after successful SES placement with RA for severely calcified lesions under the guidance of IVUS using high-pressure ballooning, SES was unrelated to the primary endpoint (Table II), and the low incidence ratio of primary endpoint (only 2.3\%) with the mean observational interval of 1289 days (approximately 3.5 years) (Table I) was acceptable. We followed-up the patients and lesions for the longest interval after SES placement with RA for calcified lesions compared to previous reports, ${ }^{4,7-9,29)}$ and there was no incidence of definite ST in the SES group with a mean stent length of $47.9 \mathrm{~mm}$ (Table I). None of the definite ST could be attributed to the low incidence of a primary endpoint in the SES group because the cumulative incidences of definite ST after DES implantation in western countries were up to approximately $0.74 \%$ at 30 days and $1.5 \%$ at 4 years. ${ }^{30,31)}$ Thus, multiple long SES placement for calcified lesions with RA in our daily practice showed promising longterm safety in Japan.

The second goal of the present study was to evaluate the angiographic outcomes after SES and BMS implantations to severely calcified lesions using RA on the basis of the longterm safety after the widespread use of SES as discussed above. This was because of the possibility that SES could not exert its major benefit: ie, a better angiographic outcome at chronic phase compared to BMS in severely calcified lesions due to the following reasons: (i) vascular biological response in heavily calcified lesions could not be fully suppressed by sirolimus, ${ }^{32)}$ (ii) the suboptimal stent expansion and apposition 
due to the rigid closed cell based platform of SES in the calcified lesions, ${ }^{33)}$ and (iii) polymer damage due to delivery through the hard plaque in the calcified lesions. ${ }^{4,34}$ As a result of the present retrospective study SES did not improve the angiographic outcomes with the high BR ratio and large mean value of late luminal loss (Tables IV, V). The BR ratio (21.3\%) (Table IV) in the SES group was higher than that in the cohorts studied in two prospective randomized studies (ranging from $3 \%$ to $6 \%) .{ }^{10,11)}$ In addition, the mean value of late luminal loss $(0.47 \mathrm{~mm})$ (Table IV) was greater than the conventional values of SES (approximately ranging from 0.10 to $0.20 \mathrm{~mm}$ ). ${ }^{2,10)}$ The ratio of type II-IV ISR (45.7\%) (Table IV) was very high compared to that $(16 \%)$ of a previous report. ${ }^{11)}$ Furthermore, the incidence of severe restenosis, usually considered as the incidence of clinical target lesion revascularization, in the SES group was $10.9 \%$ and similar to that of BMS (Table IV). The large late luminal loss and the high BR ratio of SES with RA for calcified lesions can be attributed to the 3 reasons described above. In addition, the relatively high percentages of hemodialysis, the consistent predictor of both endpoints after SES placement, ${ }^{2,3,35)}$ included in the present cohort (Tables I, III, V) are related to the ineffectiveness of SES for the angiographic outcome. Accordingly, SES with RA for calcified lesions did not exert a beneficial effect at reducing the incidence of BR in the widespread indication in our daily practice. Thus, this issue that SES did not exert its benefit for angiographic outcome including the relative percentages of hemodialysis for calcified lesions using RA has to be further examined in prospective randomized studies using new-generation DES, by changing the drug, revising the comformability and flexibility of the stent platforms, and optimizing polymer properties.

Several limitations to the study should be considered. First, because this trial was a single-center, retrospective, and nonrandomized study, the confounding factors influencing the endpoints were not clear. The outcomes of SES were largely determined by a historical comparison with those of BMS. Thus, many baseline variables were different between the BMS and SES groups, reflecting the procedural differences between the pre-SES and post-SES approval eras (Tables I and III). Thus, although it was difficult to completely match the baseline variables in both groups, the subanalysis was conducted by selecting the cases of similar stent length with similar stent diameter from the cohort followed-up angiographically (Table VI) in order to further ascertain whether SES had a benefit for severely calcified lesions using RA or not. Although almost all of the procedure-related variables were similar in the two groups, with the exception of inflation pressure, the BR ratio in the SES group was not significantly lower than that in the BMS group in the present very small cohort (Table VI). Thus, as described above, these issues should be further examined in prospective and larger-scale studies in the present second-generation DES era. Second, the effect of SES on calcified lesions successfully implanted without RA was unclear. Third, since patients with former-generation BMS were mainly enrolled, the durability of SES may have been overestimated. Fourth, although the stents were mainly deployed with IVUS (Tables I, III), intravascular assessment factors such as minimal stent area and symmetric index could not be satisfactorily evaluated.

Conclusion: Although this retrospective nonrandomized historical study showed the long-term safety of SES for calcified lesions using a rotablator, SES was not associated with a benefit with respect to angiographic outcomes compared to BMS. Further prospective and larger-scale examinations in the present second-generation DES era will be needed.

\section{REFERENCES}

1. Schenker MP, Dorbala S, Hong EC, et al. Interrelation of coronary calcification, myocardial ischemia, and outcomes in patients with intermediate likelihood of coronary artery disease: a combined positron emission tomography/computed tomography study. Circulation 2008; 117: 1693-700.

2. Ikari Y, Kotani J, Kozuma K, et al. Assessment of sirolimus-eluting coronary stent implantation with aspirin plus low dose ticlopidine administration: one year results from CYPHER Stent Japan Post-Marketing Surveillance Registry (J-PMS). Circ J 2009; 73: 1038-44.

3. Mutoh M, Ishikawa T, Hasuda T, et al. Predictors of target lesion revascularization and documented stent thrombosis beyond 30 days after sirolimus-eluting stent implantation: retrospective analysis in consecutive 1,070 angiographic follow-up lesions. Circ J 2007; 71: 1328-31.

4. Mori S, Yasuda S, Kataoka Y, Morii I, Kawamura A, Miyazaki S. Significant association of coronary artery calcification in stent delivery route with restenosis after sirolimus-eluting stent implantation. Circ J 2009; 73: 1856-63.

5. Fujii K, Carlier SG, Mintz GS, et al. Stent underexpansion and residual reference segment stenosis are related to stent thrombosis after sirolimus-eluting stent implantation: an intravascular ultrasound study. J Am Coll Cardiol 2005; 45: 995-8.

6. Cavusoglu E, Kini AS, Marmur JD, Sharma SK. Current status of rotational atherectomy. Catheter Cardiovasc Interv 2004; 62: 48598. (Review)

7. Clavijo LC, Steinberg DH, Torguson R, et al. Sirolimus-eluting stents and calcified coronary lesions: clinical outcomes of patients treated with and without rotational atherectomy. Catheter Cardiovasc Interv 2006; 68: 873-8.

8. Tamekiyo H, Hayashi Y, Toyofuku M, et al. Clinical outcomes of sirolimus-eluting stenting after rotational atherectomy. Circ J 2009; 73: 2042-9.

9. Rathore S, Matsuo H, Terashima M, et al. Rotational atherectomy for fibro-calcific coronary artery disease in drug eluting stent era: procedural outcomes and angiographic follow-up results. Catheter Cardiovasc Interv 2010; 75: 919-27.

10. Moses JW, Leon MB, Popma JJ, et al. Sirolimus-eluting stents versus standard stents in patients with stenosis in a native coronary artery. N Engl J Med 2003; 349: 1315-23.

11. Popma JJ, Leon MB, Moses JW, et al. Quantitative assessment of angiographic restenosis after sirolimus-eluting stent implantation in native coronary arteries. Circulation 2004; 110: 3773-80.

12. Kaplan BM, Safian RD, Mojares JJ, et al. Optimal burr and adjunctive balloon sizing reduces the need for target artery revascularization after coronary mechanical rotational atherectomy. Am J Cardiol 1996; 78: 1224-9.

13. Hong MK, Mintz GS, Lee CW, et al. Intravascular ultrasound predictors of angiographic restenosis after sirolimus-eluting stent implantation. Eur Heart J 2006; 27: 1305-10.

14. Roy P, Steinberg DH, Sushinsky SJ, et al. The potential clinical utility of intravascular ultrasound guidance in patients undergoing percutaneous coronary intervention with drug-eluting stents. Eur Heart J 2008; 29: 1851-7.

15. Mutoh M, Ishikawa T, Sakamoto H, et al. Facilitation of rotablator-stenting by measuring angiographic lesion length with the navicath (type-MUTO), a new measuring microcatheter. Int Heart J 2006; 47: 139-46.

16. Romagnoli E, Sangiorgi GM, Cosgrave J, Guillet E, Colombo A. Drug-eluting stenting: the case for post-dilation. JACC Cardiovasc Interv 2008; 1: 22-31. (Review) 
17. Cutlip DE, Windecker S, Mehran R, et al. Clinical end points in coronary stent trials: a case for standardized definitions. Circulation 2007; 115: 2344-51

18. Sakamoto H, Ishikawa T, Mutoh M, et al. Effectiveness of a sirolimus-eluting stent (Cypher) for diffuse in-stent restenosis inside a bare metal stent. Int Heart J 2006; 47: 651-61.

19. Mehran R, Dangas G, Abizaid AS, et al. Angiographic patterns of in-stent restenosis: classification and implications for long-term outcome. Circulation 1999; 100: 1872-8.

20. Wexler L, Brundage B, Crouse J, et al. Coronary artery calcification: pathophysiology, epidemiology, imaging methods, and clinical implications. A statement for health professionals from the American Heart Association. Writing Group. Circulation 1996; 94: 1175-92.

21. Ramana RK, Joyal D, Arab D, et al. Clinical experience with rotational atherectomy in patients with severe left ventricular dysfunction. J Invasive Cardiol 2006; 18: 514-8.

22. Kimura T, Morimoto T, Nakagawa Y, et al. Antiplatelet therapy and stent thrombosis after sirolimus-eluting stent implantation. Circulation 2009; 24, 119: 987-95.

23. Ishikawa T, Nakano Y, Endoh A, et al. Significantly lower incidence of early definite stent thrombosis of drug-eluting stents after unrestricted use in Japan using ticlopidine compared to western countries using clopidogrel: a retrospective comparison with western mega-studies. J Cardiol 2009; 54: 238-44.

24. Brener SJ, Lytle BW, Casserly IP, Schneider JP, Topol EJ, Lauer MS. Propensity analysis of long-term survival after surgical or percutaneous revascularization in patients with multivessel coronary artery disease and high-risk features. Circulation 2004; 109: 2290-5.

25. Iakovou I, Schmidt T, Bonizzoni E, et al. Incidence, predictors, and outcome of thrombosis after successful implantation of drugeluting stents. JAMA 2005; 293: 2126-30.

26. Lemos PA, Hoye A, Goedhart D, et al. Clinical, angiographic, and procedural predictors of angiographic restenosis after sirolimus- eluting stent implantation in complex patients: an evaluation from the Rapamycin-Eluting Stent Evaluated At Rotterdam Cardiology Hospital (RESEARCH) study. Circulation 2004; 109: 1366-70.

27. Shirai S, Kimura T, Nobuyoshi M, et al. Impact of multiple and long sirolimus-eluting stent implantation on 3-year clinical outcomes in the j-Cypher Registry. JACC Cardiovasc Interv 2010; 3 : 180-8.

28. Hildick-Smith D, de Belder AJ, Cooter N, et al. Randomized trial of simple versus complex drug-eluting stenting for bifurcation lesions: the British Bifurcation Coronary Study: old, new, and evolving strategies. Circulation 2010; 121: 1235-43.

29. Khattab AA, Otto A, Hochadel M, Toelg R, Geist V, Richardt G. Drug-eluting stents versus bare metal stents following rotational atherectomy for heavily calcified coronary lesions: late angiographic and clinical follow-up results. J Interv Cardiol 2007; 20: $100-6$.

30. de la Torre-Hernández JM, Alfonso F, Hernández F, et al. Drugeluting stent thrombosis: results from the multicenter Spanish registry ESTROFA. (Estudio ESpañol sobre TROmbosis de stents FArmacoacti vos.) J Am Coll Cardiol 2008; 51: 986-90.

31. Stettler C, Wandel S, Allemann S, et al. Outcomes associated with drug-eluting and bare-metal stents: a collaborative network metaanalysis. Lancet 2007; 370: 937-48. (Review)

32. Costa MA, Simon DI. Molecular basis of restenosis and drugeluting stents. Circulation 2005; 111: 2257-73. (Review).

33. Tanigawa J, Barlis P, Mario C. Heavily calcified coronary lesions preclude strut apposition despite high pressure balloon dilatation and rotational atherectomy: in-vivo demonstration with optical coherence tomography. Circ J 2008; 72: 157-60.

34. Kuriyama N, Kobayashi Y, Nakayama T, Kuroda N, Komuro I. Images in cardiovascular medicine. Damage to polymer of a sirolimus-eluting stent. Circulation 2006; 114: e586-7.

35. Ichimoto E, Kobayashi Y, Iijima Y, Kuroda N, Kohno Y, Komuro I. Long-term clinical outcomes after sirolimus-eluting stent implantation in dialysis patients. Int Heart J 2010; 51: 92-7. 